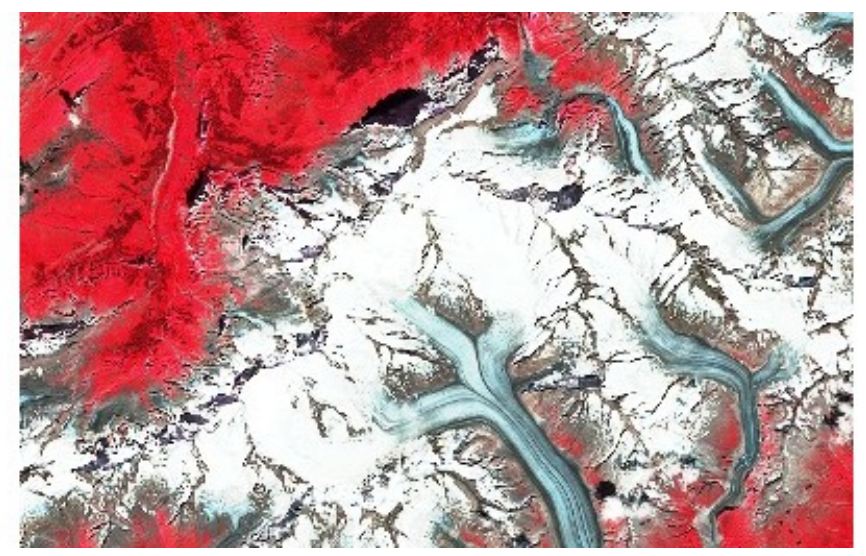

\title{
A Covid-19 death trail connecting the Mediterranean with the North Sea, Italy with England, through the Alps
}

\author{
Carlos Rodriguez 1 \\ 1 Fundación Jiménez Díaz
}

Funding: The author(s) received no specific funding for this work.

Potential competing interests: The author(s) declared that no potential competing interests exist.

\section{Abstract}

By displaying Covid-19 mortality data at a subnational level I show here a surprising distribution pattern, one that draws a straight line of high mortality areas that transversally crosses central Europe, linking together northern Italy with central/east England.

The end of winter 2020 was a scary time across Europe. A new disease was hitting merciless right and left, overwhelming well equipped ICUs in several big countries. In the blink of an eye, apparently healthy people, were suddenly having trouble breathing. Some of them ending in an ICU, and dying of a terrible death: asphyxiating. It was all over Europe. Or was it?

At the time, you would hear in the news that countries like Italy and Spain were registering unbearable death tolls, then France, then Belgium and the UK. Media were repeating mantras like "Germany must be doing something much better that us since their death toll is comparatively so low" or "What is going on with Belgium? Is their Health System collapsing?" We were running long of words and short of true explanations for that unexpected blow to a whole continent.

So far for the first part of this story. 
The second part relates more to the way we interpret numbers during a pandemic. Since this was a first for most of us, we had to quickly get used to the likes of reproduction numbers, herd immunity, herd immunity threshole, death rate, seroprevalence, dispersion, contact tracing or lockdowns. New/old terms in a cocktail of words that often times were adding confusion rather than clarity to the whole picture.

Now that the death toll has receded in most western Europe, it is time to look back at those confusing moments and try to shed some light on the numbers. Some of those numbers still haunt me as I have a hard time finding true meaningfulness in them. In fact, after thoroughly studying the data I had to conclude that, when trying to understand the whole dynamics of the pandemic, most of these data is meaningless. This is clearly the case for terms like number of new cases (what does it mean: number of new PCR+, number of new symptomatic/asymptomatic patients, number of recurrences?), or the cummulative death toll by country. This latter is particularly confusing, since it refers to naked data devoided of its population context. For instance, if we only look at number of deaths we get countries like India on top of the list. However, when we considerer a little finer statistics like deaths per million people (dpm), we then find India at the bottom of the list, since with $40 \mathrm{dpm}$, it has a mortality rate 15 times lower than the US, UK or Spain. In summary, dpm is more meaningful because what really matters in this disease is the people who die, not the people who get infected (i.e. PCR+ $)^{[1]}$.

But for the sake of refining statistics, showing $d p m$ by country is still not enough. In fact, it gives us a too broad of a picture of the pandemic, without showing us the nuances that one could obtain with yet more refined maps. For instance, the map in figure $1 \mathrm{a}$ is telling us, among other things, that Portugal has a much lower death rate than Spain. But, by now, we know that this is not the whole truth, since when we display $d p m$ by smaller geografic areas (GA), like provinces, a whole different picture emerges (Fibure 1B), one that shows a much lower death rate not only in Portugal but on the whole Atlantic belt of the Iberian Peninsula as well as the southern Mediterranean coast of Spain. Contrary to the narrow view delivered by countrywise statistics, displaying data on a subnational level uncovers patterns that otherwise could go unappreciated. This same observation on the whole Atlantic Europe has previously allow me to speculate that weather phenomena, like for instance the Gulf Stream, could somehow determine the outcome of the disease in a whole continent ${ }^{[2]}$. 

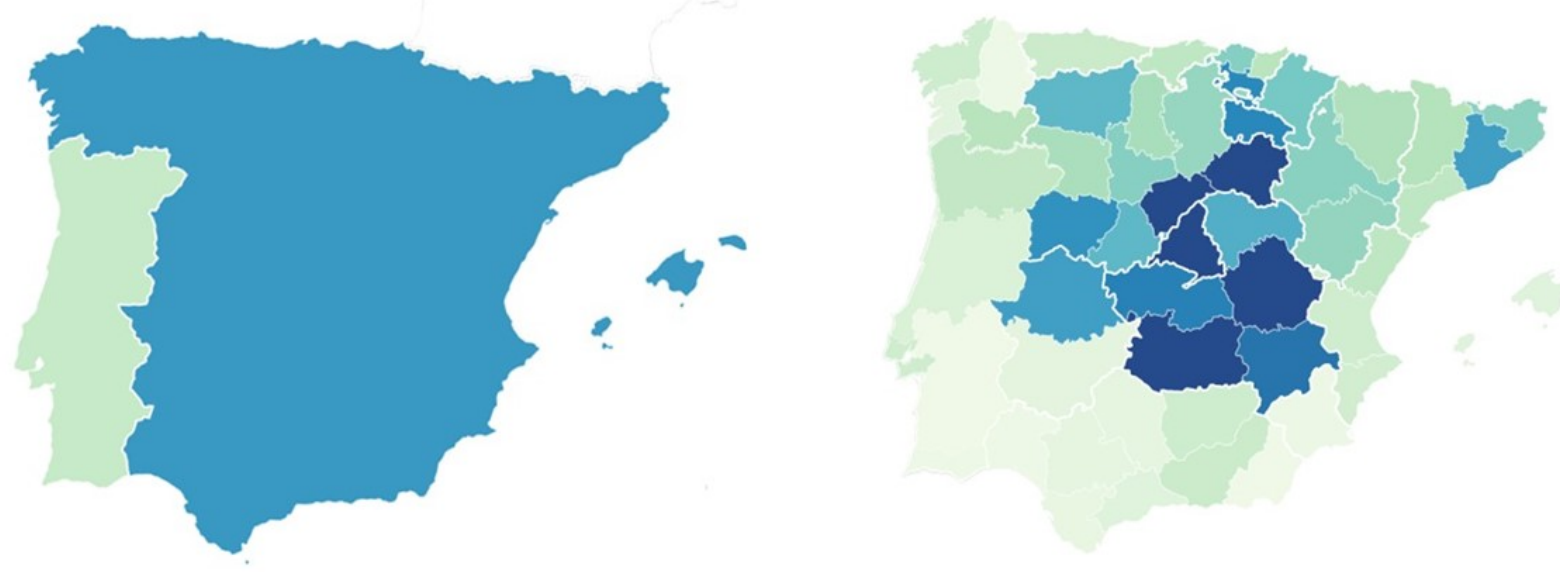

A

B

Figure 1 Maps of Covid-19 dpm by country (A) or by provinces (B). Different huyes of green represent Covid-19 lower mortality rate areas

In order to uncover more patterns in other European GA, I applied the same approach to central Europe. When we do this, again, a new pattern emerges (Figure 2B). In this new picture of Europe, we can easily make a couple of striking observations. 


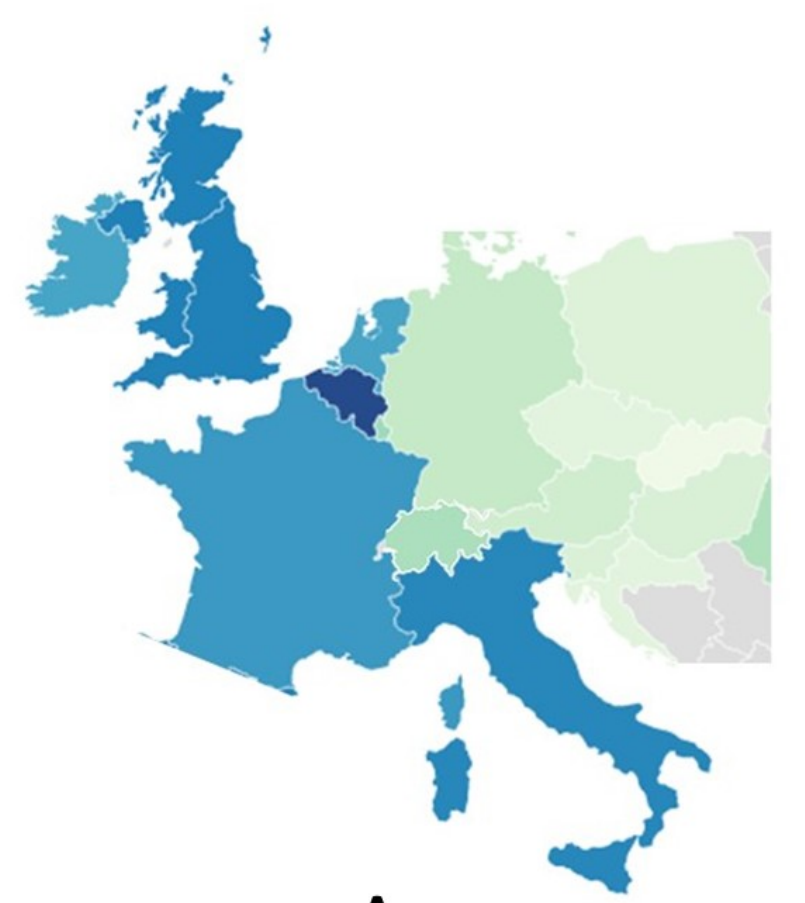

A

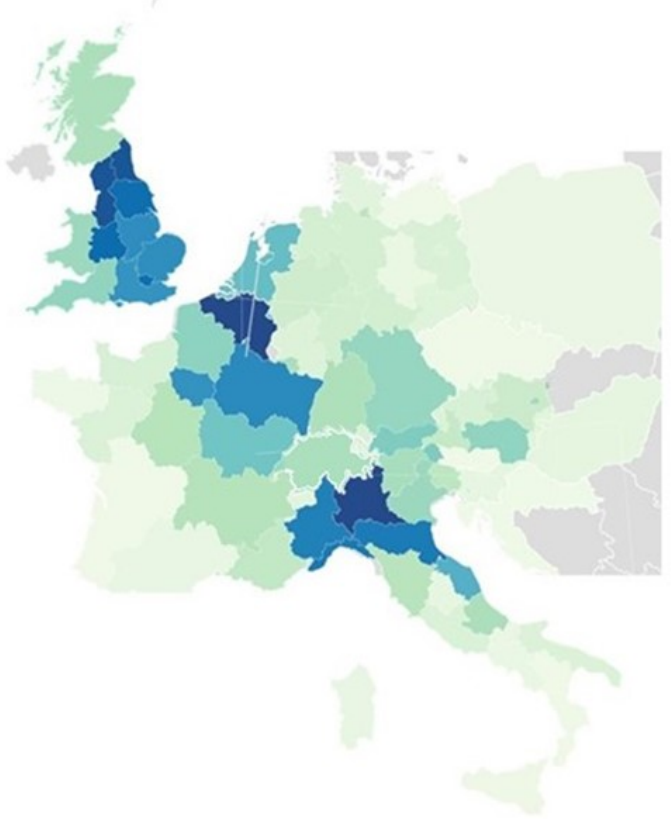

B

Figure 2 A. Covid-19 mortality (dpm) by country (Blue color represents areas with Covid-19 mortality rates higher than $400 \mathrm{dpm}$ ). B. Covid-19 mortality by region.

First, there seems to be a corridor of GAs with high mortality rate that runs from Northern Italy, through the Alps, crossing Northern France and Belgium, and finally reaching central and east England (Figure 2B). Put in other words, a straight line connecting the Mediterranean (i.e. the Adriatic Sea) with the North See can be drawn uninterrumptedly through this trail (figure 3), signaling a unique phenomenon that begs an explanation. 


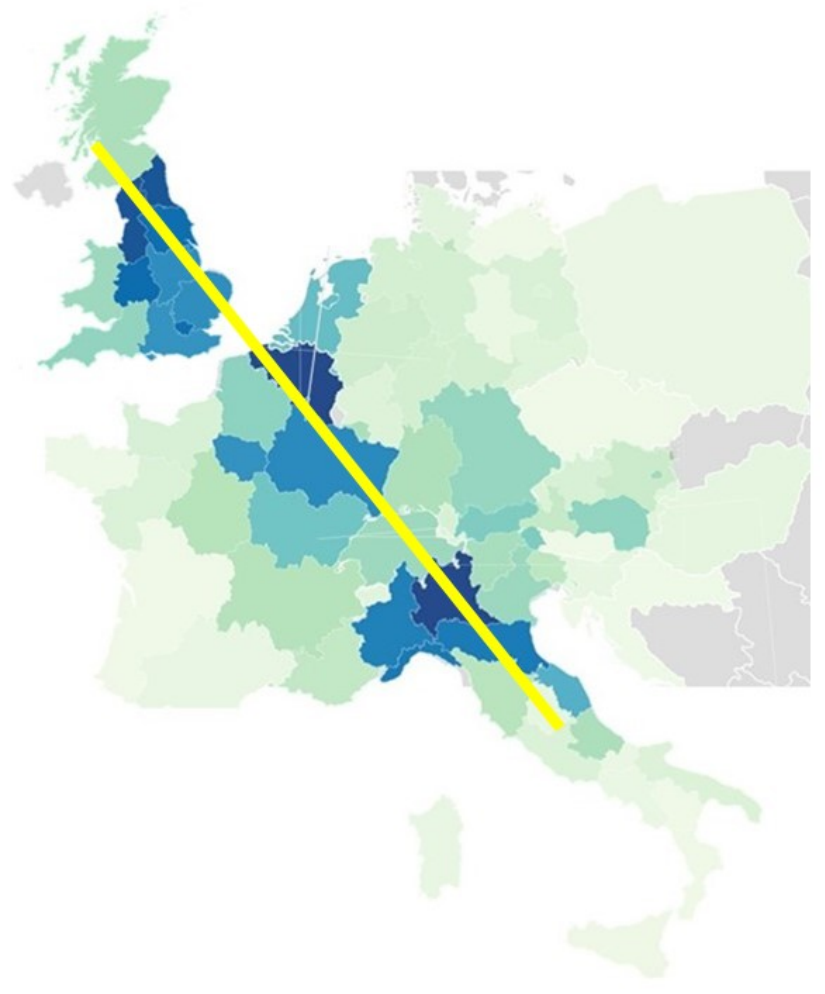

Figure 3

Second, on both sides of the trail there appears to be a gradient of progressively decreasing mortality. This can be more clearly appreciated on the French side of the axis where data are displayed by regions (figure 3).

I am not a weather scientist nor a geologist, so I won't even try to find a thorough scientific explanation for this phenomenon. What I can do however is to throw a few hypothesis to spark something of a preliminary discussion.

I will first mention the Foehn winds. Foehn is a collective term for warm (upslope) and dry downslope winds. Foehn winds are found in many mountains around the world. In the Alps, they are named depending on the direction the wind comes from. In south foehns, for instance, humid air from the Mediterranean sea ascends the slopes of the southern Alps ${ }^{[3]}$. A place were these winds are particularly strong is in Ticino, southern Switzerland-northern Italy, not surprisingly the region with the highest mortality rate by Covid-19. Interestingly, back in February 2020, just days before the first cases were diagnosed in northern Italy, this wind was especially persistent and, quoting a local meteoweb, "the foehn was king for a good part of the month: there were 8 favonic days, locally 10. In particular, on February 5th the föhn was really very intense: the gusts recorded were between 50 and $90 \mathrm{~km} / \mathrm{h}$ with a peak in Gera Lario of $140 \mathrm{~km} / \mathrm{h}$ and over $100 \mathrm{~km} / \mathrm{h}$ on the pre-alpine reliefs" (https://www.meteobassacomasca.it/febbraio-2020-secco-mitezza-e-favonio). Is it possible, then, that cyclonic winds heading into the Alps from the Mediterranean Sea could have somehow shaped the first chronology of the pandemic in Nordern Italy? In fact, we could easily imagine these winds starting on the Adriatic coast of the Italian Marche region, advancing north through Emilia-Romagna, and finally reaching Lombardia and the Alps. This imaginary wind gust will almost perfectly overlap with a gradient like death rate map starting on the coasts of Marche and ending on 
the shores of lake Cuomo, Lombardia, which, together with Swiss Ticino, were hit by Covid-19 at its heaviest (figure 4). Whether southern foehns continue influencing the regions north-west of the Alps, or, whether other winds/currents originating in the north slopes of the mountain chain could be linked with the covid-19 death trail towards England is up for discussion/investigation, and, therefore, I leave it here for the experts to continue delving into the problem.
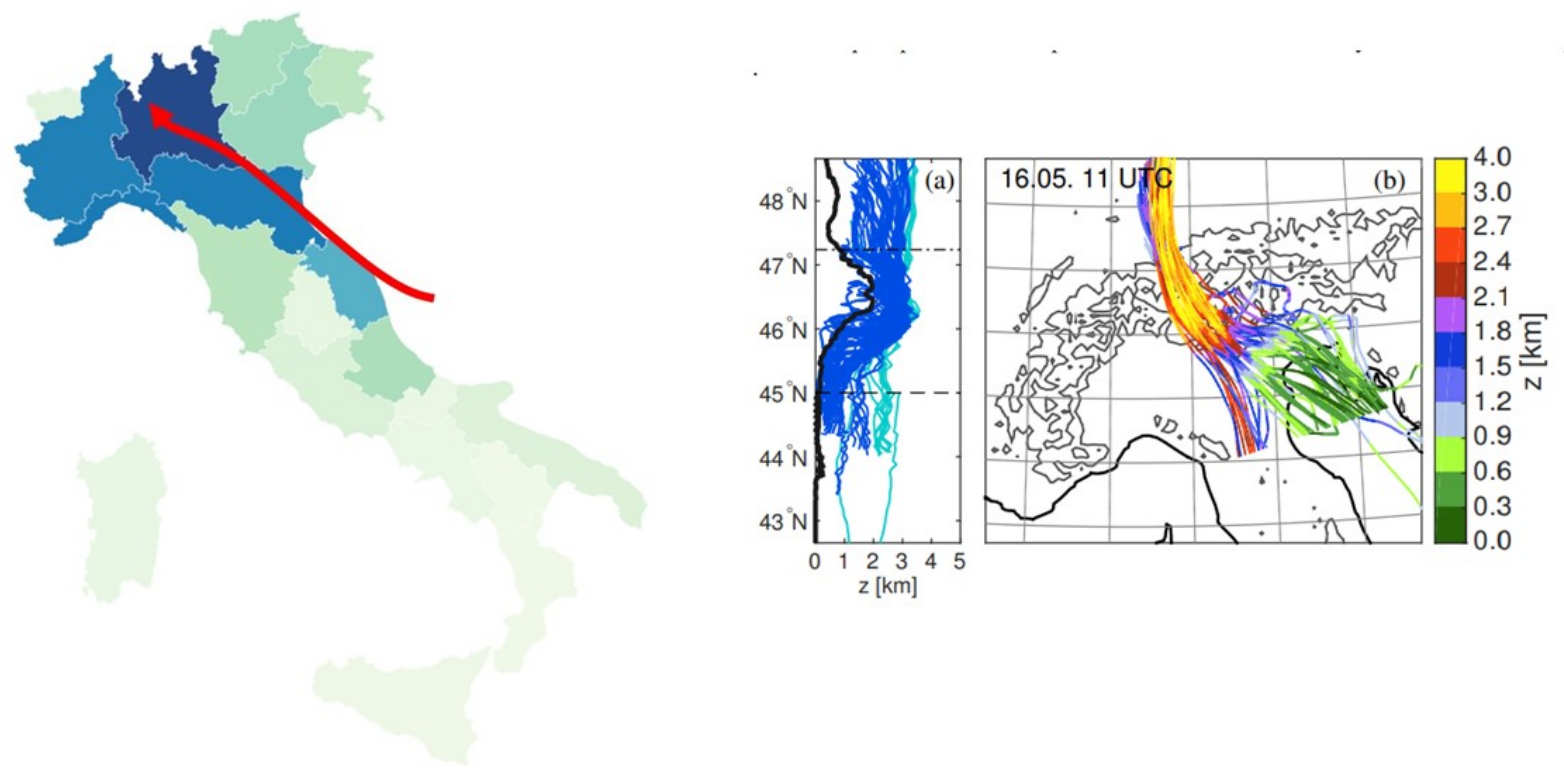

A

B

Figure 4. A Map of Italy showing in blue the higher death rate areas. The red arrow depicts the likely course of the Adriatic foehn. B. Langrarian analysis of foehn events over the alps from Miltemberger et al. ${ }^{[3]}$

Additionally, other observations could provide some explanatory insight into the death trail phenomenon. For instance, it has been shown that areas with high levels of nitrogen dioxide in the troposphere overlap with some of the geographic areas with highest death rates by Covid-19, including northern Italy and central Spain ${ }^{[4]}$.

All in all, I have shown here how, by displaying the same data in a different way, we can uncover patterns related to the geographic impact of the disease that otherwise would go completely undetected.

To summarize:

1. The most meaningful data to understand geographical distribution patterns in the Covid-19 pandemic are those expressed in number of deaths par capita, which provide a more clear picture of the impact of the pandemic on whole populations.

2. Ideally maps should show as local as possible data, at the region, province, county or, even better, at the municipality 
level. Hopefully, by doing that, we would be able to obtain an even more precise picture of the data, one that allow us to model weather or geographic patterns associated with the covid-19 pandemic and make predictions accordingly.

3. After charting these new data, I have uncovered an East-West crest of Covid-19 high mortality rate that crosses central Europe as a continuous straight line from Italy to England. Whether Alpine driven meteorology or other atmospheric processes are linked to the phenomenon, this should be the subject of future investigation by experts in the related fields.

\section{References}

1. ^ Carlos Rodriguez. (2020). SETTING THE PRIORITIES RIGHT IN THE COVID-19 PANDEMIC. Qeios. doi:10.32388/TSL2KJ.

2. `Carlos Rodriguez. (2020). Gulf Stream gyres may have dictated the outcome of the Covid-19 disease in Western Europe. Qeios. doi:10.32388/ME7VKG.

3. ${ }^{\mathrm{a}, \mathrm{b}}$ Annette K. Miltenberger, Silvia Reynolds, Michael Sprenger. (2016). Revisiting the latent heating contribution to foehn warming: Lagrangian analysis of two foehn events over the Swiss Alps. Q.J.R. Meteorol. Soc., vol. 142 (698), 2194-2204. doi:10.1002/qj.2816.

4. ' Yaron Ogen. (2020). Assessing nitrogen dioxide (NO2) levels as a contributing factor to coronavirus (COVID-19) fatality. Science of The Total Environment, vol. 726 , 138605. doi:10.1016/j.scitotenv.2020.138605. 